\title{
Combination of HSP90 and autophagy inhibitors promotes hepatocellular carcinoma apoptosis following incomplete thermal ablation
}

\author{
FEN CHEN ${ }^{1}$, HAIYANG XIE ${ }^{2}$, HAIWEI BAO ${ }^{1}$, LAURENCIA VIOLETTA $^{3}$ and SHUSEN ZHENG ${ }^{4}$ \\ ${ }^{1}$ Department of Ultrasound, The First Affiliated Hospital of Zhejiang Chinese Medical University; \\ ${ }^{2}$ Key Laboratory of Combined Multi-organ Transplantation, Ministry of Public Health, \\ Division of Hepatobiliary and Pancreatic Surgery, First Affiliated Hospital, School of Medicine, \\ Zhejiang University; ${ }^{3}$ State Key Laboratory for Diagnosis and Treatment of Infectious Disease; \\ ${ }^{4}$ Division of Hepatobiliary and Pancreatic Surgery, Department of Surgery, First Affiliated Hospital, School of Medicine, \\ Zhejiang University, Hangzhou, Zhejiang 310002, P.R. China
}

Received June 1, 2019; Accepted February 24, 2020

DOI: $10.3892 / \mathrm{mmr} .2020 .11080$

\begin{abstract}
The present study evaluated the effect of combining inhibitors (17-AAG) of heat shock protein 90 (HSP90) and autophagy (3-MA) on apoptosis using an incomplete thermal ablation animal model. A total of 28 orthotopic mice with hepatocellular carcinoma were randomly divided into 4 groups to receive different drug interventions. Following palliative laser ablation, changes in autophagy, apoptosis and Akt/mTOR expression levels were assessed in tumors. Compared with the controls, the 17-AAG-treated mice exhibited significantly decreased expression levels of phosphorylated (p)-Akt and p-mTOR with enhanced autophagy and apoptosis; no marked increases in the expression levels of p-Akt and p-mTOR were observed in the 3-MA-treated mice, with no significant changes in autophagy; however, apoptosis was enhanced. No significant decreases in p-Akt and p-mTOR or any increase in autophagy were observed in the mice receiving a combination of 17-AAG and 3-MA, but they did exhibit a marked increase in apoptosis. Compared with 17-AAG alone, the combination of 17-AAG and 3-MA resulted in a marked increase in apoptosis without enhanced autophagy. In the incomplete ablation model, the effects of autophagy and apoptosis are antagonistic. The combined use of 17-AAG and 3-MA can significantly promote apoptosis and is worthy of further study.
\end{abstract}

Correspondence to: Dr Shusen Zheng, Division of Hepatobiliary and Pancreatic Surgery, Department of Surgery, First Affiliated Hospital, School of Medicine, Zhejiang University, 79 Qingchun Road, Hangzhou, Zhejiang 310002, P.R. China

E-mail: shusenzheng@zju.edu.cn

Key words: hepatocellular carcinoma, thermal ablation, apoptosis, autophagy, heat shock protein 90

\section{Introduction}

In addition to being minimally invasive, safe, effective and amenable to repeated treatments, thermal ablation has been demonstrated to produce satisfactory long-term follow-up results in the treatment of hepatocellular carcinoma (HCC) and is considered an important radical curative therapy for this disease (1). Due to the effect of the ablation mechanism, the pathological characteristics of liver cancer and the anatomical characteristics of the liver, a certain proportion of the residual tumor remains even following ablation therapy, which is the main factor affecting the survival rate of patients with HCC following ablation $(2,3)$.

New strategies based on an improved understanding of residual tumor biology may help to maximize the efficacy of current treatments. There has been steadily increasing interest in changes in heat shock protein (HSP) expression and its antagonistic effect on apoptosis (4-8). The application of HSP90 inhibitors to improve the therapeutic effect on tumors has also been explored in several studies (9-17). However, due to the regulatory effects and complex downstream molecular changes HSPs exert on numerous companion proteins (14), current research on the treatment of advanced tumors remains limited.

The autophagy system serves multiple roles in the degradation of dysfunctional proteins. In the case of incomplete ablation, we first reported the decreased expression of autophagy-associated proteins and a concomitant increase in the expression of HSPs (18). HSP90, as a molecular chaperone, is essential for the proper function of Akt as it forms a chaperone-substrate protein complex, and a decrease in HSP90-Akt binding results in Akt inactivation (19-21). The activation of AKT is characterized by high levels of Akt phosphorylation (p-Akt), resulting in activation of the mTOR complex. mTOR acts as a major checkpoint regulating autophagy (22). Further, it has been suggested that the HSP90/Akt/mTOR pathway is involved in signal transmission between autophagy and HSPs. Autophagy can either protect against or promote 
apoptosis in a stimulus-dependent manner (23-25). The relationship between the two in the incomplete ablation model is unclear. Specifically, it is unclear whether the use of autophagy inhibitors promotes apoptosis or if there a synergistic effect when autophagy inhibitors and HSP90 inhibitors are used in combination. Autophagy was assessed by analyzing the conversion of microtubule-associated protein 1A (LC3) protein from cytosolic LC3-I to the autophagosome-associated lipid-modified form LC3-II, indicative of the onset of autophagy (24). The present study hypothesized that autophagy serves an important role in apoptosis during the treatment of residual tumors with HSP90 inhibitors following incomplete ablation, and it analyzed the coordinating mechanisms mediating the interplay between these processes. The HSP90 inhibitor 17-N -allylamino-17-demethoxygeldanamycin (17-AAG), which is a derivative of the antibiotic geldanamycin, has already been used in stage III clinical trials (NCT00546780) (26) and 3-methyladenine (3-MA) has been well established as an autophagy inhibitor. This present study builds upon previous studies that explore the possibility of improving the therapeutic effect of thermal ablation by combining inhibitors of autophagy and HSP90.

\section{Materials and methods}

Animal model. A total of 28 nude mice transplanted with the Huh7 tumor cell line were randomly divided into 4 groups of 7 mice each. All model animals underwent laser ablation with different drug combination therapies. The protocol of this study was approved by the Research Ethics Committee of the First Affiliated Hospital, College of Medicine, Zhejiang University. In brief, healthy female nude mice (body weight 12-16 g; 4 weeks of age; $n=28$ ) were purchased from the Shanghai Cancer Institute. The nude mice were housed in a specific pathogen-free (SPF) animal room at a temperature of $20-25^{\circ} \mathrm{C}$, humidity of $50-60 \%$, and were subjected to a 12-h light/dark cycle with free access to food and tap water. All experimental procedures followed the operational specifications for experimental animals at Zhejiang University (http://www.lac.zju.edu.cn/cms/12997). Huh7 tumor tissue exhibiting sufficient growth up to $10 \mathrm{~mm}$ in length was cut into $2-\mathrm{mm}^{3}$ pieces and subcutaneously implanted into the right flanks of nude mice using a 20-gauge trocar. A total of 28 mice were used for the experiments after 3 weeks, when tumors had grown to an average diameter of $0.8-1.0 \mathrm{~cm}$. The grouping was performed using the random number method. On the day of ablation, the average diameter of the tumors was $1.0-1.2 \mathrm{~cm}$ and average volume of tumors was $617.9 \pm 138.7 \mathrm{~mm}^{3}$. The largest tumor diameter was $1.2-1.4 \mathrm{~cm}$ and multiple tumors were not present at the end of the experiment.

Palliative ablation method. Intraperitoneal anesthesia was administered to the nude mice in the experimental and control groups with approximately $200 \mu 14 \%$ chloral hydrate at a dose of $400 \mathrm{mg} / \mathrm{kg}$ mouse weight. The conditions established in a previous study were applied to build a model of incomplete ablation (18). Mice were fixed on the operating table with local disinfection and the tumor size was observed and measured by high-frequency ultrasound. An ultrasound-guided 21G PTC needle was used to puncture the tumor and the inner core was withdrawn. Subsequently, a laser fiber was inserted into the needle $\left(\right.$ Mylab $^{\mathrm{TM}}$ Twice, Esaote SpA). After the fiber was ultrasonically confirmed to be positioned at $1 / 3$ the length of the tumor, the power was set at $1 \mathrm{~W}$ and the foot switch was activated for continuous laser ablation; the treatment time was $30 \mathrm{sec}$ (total dose of $30 \mathrm{~J}$ ). Following treatment, the fiber was removed and local hemostasis was conducted by applying gentle pressure.

Drug preparation and intravenous injection. The 17-AAG inhibitor (Selleck Chemicals LLC) was prepared by dissolving $50 \mathrm{mg}$ in $800 \mu \mathrm{l}$ DMSO solution and then diluting this to a concentration of $5 \mathrm{mg} / \mathrm{ml}$ with normal saline solution. The 3-MA inhibitor (Selleck Chemicals LLC) was prepared by dissolving $50 \mathrm{mg}$ directly in $10 \mathrm{ml}$ normal saline to produce a $5 \mathrm{mg} / \mathrm{ml}$ stock concentration. The method of drug administration was as follows: In group 1, each mouse received an intraperitoneal injection of $300 \mu \mathrm{lDMSO}$ (concentration $0.8 \%$ ); in group 2, each mouse received an intraperitoneal injection of $200 \mu \mathrm{l} 17-\mathrm{AAG}$ (concentration $5 \mathrm{mg} / \mathrm{ml}$ ); in group 3, each mouse received an intraperitoneal injection of $300 \mu \mathrm{l} 3$-MA (concentration $5 \mathrm{mg} / \mathrm{ml}$ ); in group 4, each mouse received an intraperitoneal injection of $200 \mu 1$ 17-AAG (concentration $5 \mathrm{mg} / \mathrm{ml}$ ) and, $30 \mathrm{~min}$ later, an intraperitoneal injection of $300 \mu 13$-MA (concentration $5 \mathrm{mg} / \mathrm{ml}$ ) was administered. The drugs were administered twice, at $25 \mathrm{~h}$ and $1 \mathrm{~h}$ prior to surgery.

Sample collection. Nude mice were euthanatized via intraperitoneal injection of $2 \%$ sodium pentobarbital at a dose of $150 \mathrm{mg} / \mathrm{kg}$ body weight. Cervical dislocation was used to confirm the death of mice. The nude mice were sacrificed $24 \mathrm{~h}$ post-surgery; the tumors were then extracted and their volumes were measured. The tumors were cut along their diameter and three-quarters of each tumor was kept in a liquid nitrogen jar, whereas the remaining one-quarter was fixed in $4 \%$ paraformaldehyde for $48 \mathrm{~h}$ at room temperature.

TUNEL. TUNEL (G3250; Promega Corporation) was used to determine apoptosis in HCC model mice that underwent incomplete ablation. Previously fixed tumor tissue was dehydrated in graded ethyl alcohol (70, 80, 90 and 100\%), embedded in paraffin, sliced into $4 \mu \mathrm{m}$ sections, and rehydrated $(100,95,85$ and $75 \%)$. The slides were immersed in $4 \%$ formaldehyde in PBS for $15 \mathrm{~min}$ and then in PBS for 5 min. Equilibration buffer $(100 \mu \mathrm{l})$ was added and the slides equilibrated at room temperature for 5-10 min.50 $\mu \mathrm{l}$ TdT reaction mix was added to the pretreatment slide on an area $\leq 5 \mathrm{~cm}^{2}$ for $60 \mathrm{~min}$ at $37^{\circ} \mathrm{C}$ in a humidified chamber protected from light. Reactions were stopped by immersing slides in $2 \mathrm{X}$ saline-sodium citrate buffer (15 min at room temperature). Slides were washed 3 times for 5 min each in PBS to remove unincorporated fluorescein-12-dUTP. Then, mounting medium was added to the slides. To visualize the nuclei, Vectashield with DAPI (Vector Laboratories, Inc.; Maravai LifeSciences) was used. Samples were analyzed immediately by fluorescence microscopy (Olympus Corporation). For each slide, 5 high-power fields (magnification, x200) with $>500$ cells were observed. ImagePro Plus 6.0 (Media Cybernetics, Inc.) was used to calculate the number of apoptotic cells. The apoptosis index (AI) was calculated by counting the number 
A

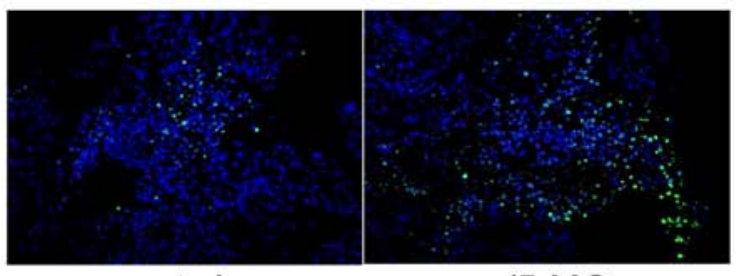

control

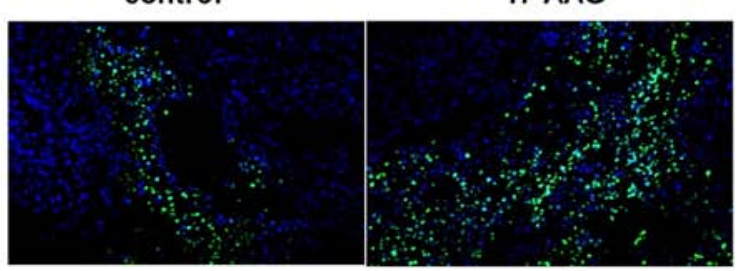

3-MA

\section{C}
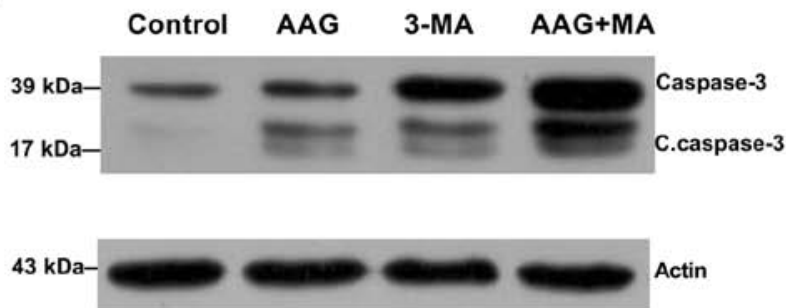

B
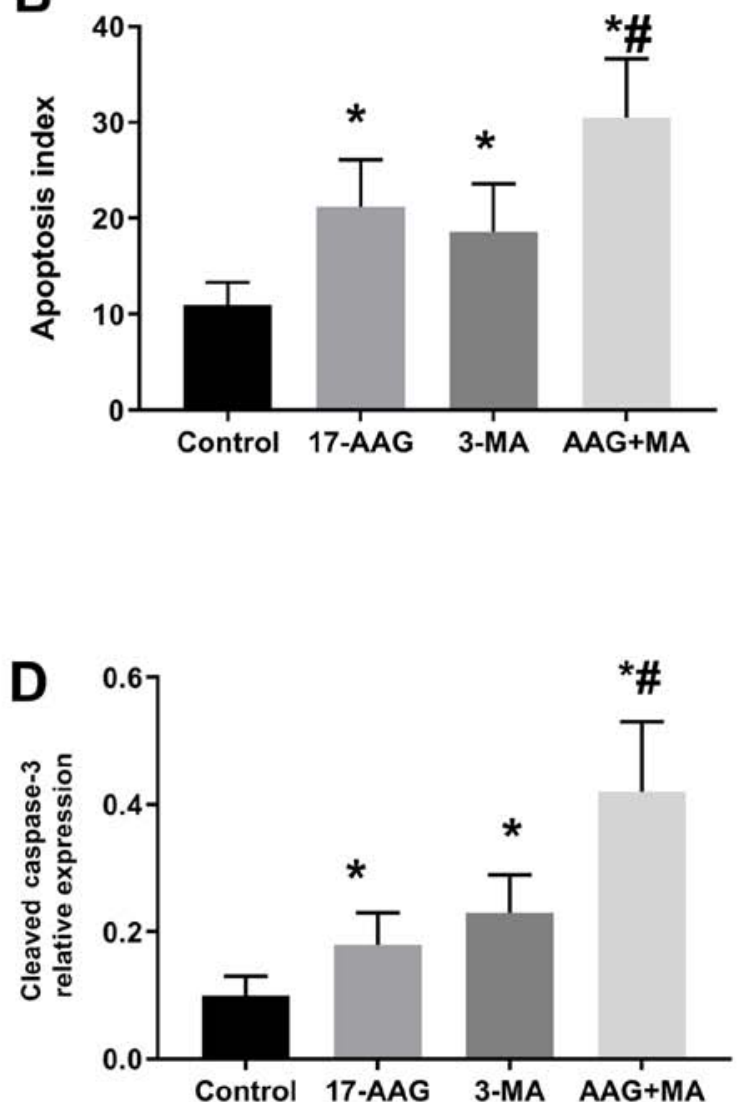

Figure 1. Apoptosis expressions in 4 groups. (A) TUNEL expression in the four groups (magnification, x200). (B) Apoptosis index of the four groups. (C) Protein levels and (D) quantitative analysis of cleaved caspase-3. The data are presented as the mean \pm standard deviation. ${ }^{*} \mathrm{P}<0.05$ vs. control; ${ }^{*} \mathrm{P}<0.05$ vs. AAG group. 17-AAG, 17-N-allylamino-17-demethoxygeldanamycin; 3-MA, 3-methyladenine.

of cells that emitted green fluorescence using the following formula:

$\mathrm{AI}=($ number of positive cells/total cell count) $\times 100 \%$.

Western blot analysis. The small tumor samples were lysed on ice using RIPA buffer containing a protease inhibitor cocktail with constant shaking for $30 \mathrm{~min}$; cellular debris was pelleted by centrifugation at $10,000 \mathrm{x} \mathrm{g}$ for $5 \mathrm{~min}$ at $4^{\circ} \mathrm{C}$ and supernatants were harvested. Protein concentrations were measured by BCA protein assay kit (ab207007; Abcam). Target proteins (30 $\mu \mathrm{g}$ per lane) were separated via SDS-PAGE. Following separation with different concentrations of acrylamide gel (6\% for mTOR; $10 \%$ for AKT and P62; 12\% for caspase-3 and cleaved caspase-3; $15 \%$ for LC3II/I), the proteins were transferred onto PVDF membranes. The membrane containing the proteins was successively incubated in blocking buffer (overnight at $\left.4^{\circ} \mathrm{C}\right)$, with a primary antibody $\left(37^{\circ} \mathrm{C}\right.$ for $\left.1 \mathrm{~h}\right)$ and with a secondary antibody $\left(37^{\circ} \mathrm{C}\right.$ for $\left.1 \mathrm{~h}\right)$. The following primary antibodies were used: Anti-LC3II/I (1:200, 12741), anti-p-Akt (1:400, 4060s; both from Cell Signaling Technology, Inc.); anti-Akt (1:400, ET1609-47), anti-mTOR (1:400, ET1608-5), anti-Caspase-3 (1:200, ER30804) and anti-p62 (1:200, R1309-8) (all from Hangzhou HuaAn Biotechnology Co., Ltd.); and anti-p-mTOR (1:400, ab52757; Abcam); anti-actin (1:400, ab179467; Abcam). HRP-conjugated goat anti-mouse IgG H\&L (1:5,000, ab205719; Abcam) was used as the secondary antibody. Chemiluminescence detection was achieved by exposure to film in a darkroom. Following development and fixation with washing buffer at $20-25^{\circ} \mathrm{C}$ for 10 min (P0019, Beyotime Institute of Biotechnology), the film was photographed by FluorChem HD2 (ProteinSimple). The densities of the protein bands were determined using Image J software (v1.46; National Institutes of Health), normalized to actin expression and quantified using Microsoft Excel software (version 2016, Microsoft Corporation).

Statistical analysis. All data were analyzed using SPSS 17.0 software (SPSS Inc.). Data are expressed as the mean \pm standard deviation. The differences among different groups were examined by one-way analysis of variance followed by Tukey's post hoc test. $\mathrm{P}<0.05$ was considered to indicate a statistically significant difference.

\section{Results}

Combined use of 17-AAG and 3-MA significantly promotes apoptosis. In Fig. 1A, compared with group 1, groups 2-4 showed green fluorescent protein-LC3 accumulation. The apoptosis indices in groups 1 to 4 were $11.0 \pm 2.3,21.2 \pm 4.9$, $18.6 \pm 5.0$ and $30.5 \pm 6.1 \%$, respectively (Fig. 1B). Differences for all groups were statistically significant compared with the results of Group $1(\mathrm{P}<0.05)$ and there was a significant difference between groups 2 and $4(\mathrm{P}<0.05)$. The relative 
A

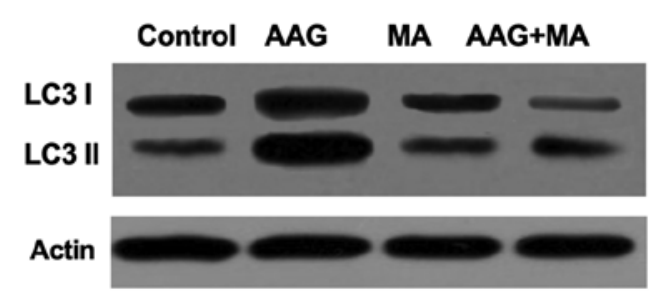

B

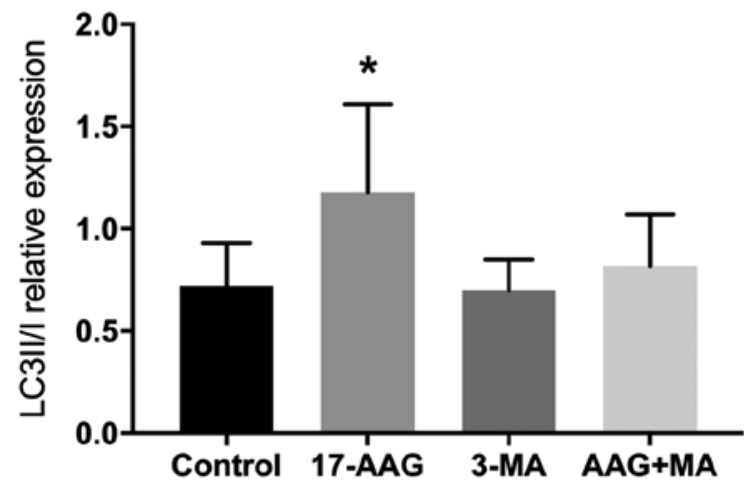

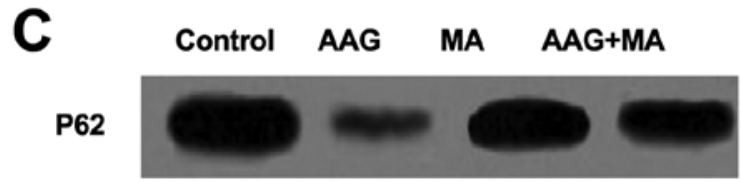
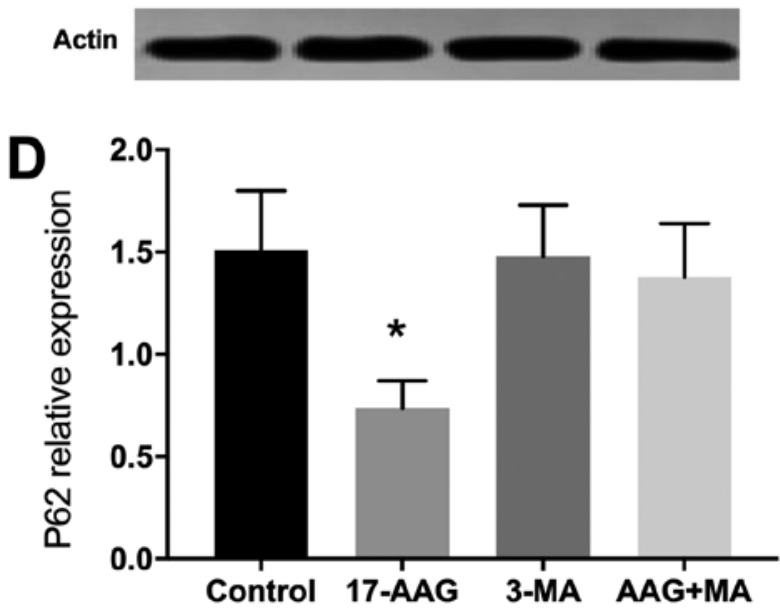

Figure 2. Autophagy in the four groups. (A) Protein levels and (B) quantitative analysis of LC3-II/I. (C) Protein levels and (D) quantitative analysis of p62. The data are presented as the mean \pm standard deviation. ${ }^{*} \mathrm{P}<0.05$ vs. control. LC3, microtubule-associated protein 1A; LC3-II, lipid modified LC3; LC3-I, 17-AAG/AAG, 17-N-allylamino-17-demethoxygeldanamycin; 3-MA/MA, 3-methyladenine; p62, protein sequestosome-1.

quantities of cleaved-capase-3 protein detected by western blot analysis in groups $1-4$ were $0.10 \pm 0.03,0.18 \pm 0.05,0.23 \pm 0.06$ and $0.42 \pm 0.11$ (Fig. 1C), respectively, with a statistically significant difference between group 1 and the other three groups (Fig. 1D). The expression of apoptosis proteins was highest in Group 4 and there was a significant difference between groups 2 and 4 (Fig. 1).

17-AAG-treatment significantly enhances autophagy. Western blot analysis of LC3 II/I expression revealed that the protein levels in groups $1-4$ were $0.72 \pm 0.21,1.18 \pm 0.43,0.70 \pm 0.15$ and $0.82 \pm 0.25$, respectively, and were significantly different between groups 1 and $2(\mathrm{P}<0.05)$. The relative protein expression levels of p62 in groups $1-4$ were $1.51 \pm 0.29,0.74 \pm 0.13$, $1.48 \pm 0.25$ and $1.38 \pm 0.26$, respectively, and were significantly different between groups 1 and 2 ( $\mathrm{P}<0.05$; Fig. 2).

17-AAG treatment significantly decreases the expression levels of $p$-Akt and $p$-mTOR. The quantities of $\mathrm{p}$-Akt relative to Akt levels in groups 1-4, as measured by western blot analysis, were $1.11 \pm 0.15,0.32 \pm 0.09,1.01 \pm 0.17$ and $1.05 \pm 0.15$, respectively. A significant difference was observed between groups 2 and $1(\mathrm{P}<0.05)$. The relative expression of $\mathrm{p}-\mathrm{mTOR}$ in groups $1-4$ was $0.58 \pm 0.12,0.32 \pm 0.09,0.67 \pm 0.14$ and $0.65 \pm 0.16$, respectively. A significant difference between groups 2 and 1 was revealed $(\mathrm{P}<0.05)$. The relative expression of mTOR was $0.81 \pm 0.19,0.75 \pm 0.21,0.85 \pm 0.23$ and $0.80 \pm 0.25$, respectively (17-AAG vs. control, P>0.05; Fig. 3).

\section{Discussion}

The present study demonstrated that suppressing autophagy enhanced HSP90 inhibitor-induced apoptosis in residual tumors. These results clearly indicated that autophagy provided a pro-survival function following incomplete ablation.
Furthermore, the combination of inhibitors targeting these 2 pathways could potentially kill residual tumor cells more effectively, thereby providing a rationale for combining these drugs as a treatment strategy for incomplete ablation. Finally, the results clearly suggested that there was an association between changes in autophagy and regulation of the Akt/mTOR signaling pathway.

The HSP90 inhibitor 17-AAG has already been used in stage III clinical trials (NCT00546780) (26). The results from stage I/II/III clinical trials of 17-AAG reveal limitations on its efficacy for the treatment of advanced tumors and particularly in allowing tumor cells to enter a quiescent phase (NCT00103272, NCT00098423, NCT00118092 and NCT001048897) (27-30). To achieve the optimum therapeutic effect for this oncotherapy, 17-AAG needs to be administered long-term or be considered for combined treatment with other anti-cancer regimens (NCT00103272) (27).

Previous studies have investigated on the combination of various drugs with HSP90 inhibitors. Lang et al (14) reported that an HSP90 inhibitor increases the efficacy of rapamycin against HepG2 and Huh7 cells by inhibiting rapamycin-induced Akt and NF-kB activation, decreasing the expression of platelet-derived growth factor receptor $\beta$ in vascular smooth muscle cells and vascular endothelial growth factor 2 expression in the vascular endothelium. Another study on non-small cell lung cancer cell lines by Webber et al (15) indicated that combining an HSP90 inhibitor (17-AAG) and a focal adhesion kinase inhibitor (PF-573228) suppresses the Akt-mTOR pathway, consequently inhibiting colony formation and promoting the activation of apoptosis-inducing proteins. Furthermore, Yang et al (16) describes the inhibition of HSP90 expression and enhancement of apoptosis using Thy-1 membrane glycoprotein (Thy-1)-targeted thermosensitive magnetoliposome-encapsulated 17-AAG for Thy-1 + liver cancer stem cells (LSCSs) selected from the BEL-7404 cell line and in a nude mouse model transplanted with Thy-1 + LCSCs tumors. 
A
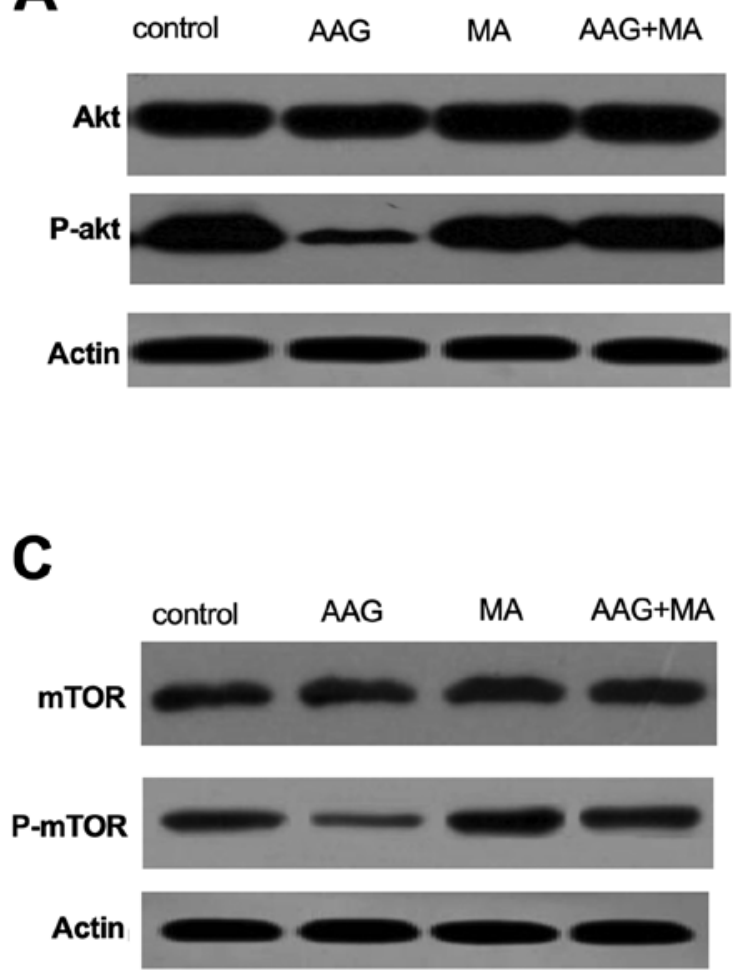

B

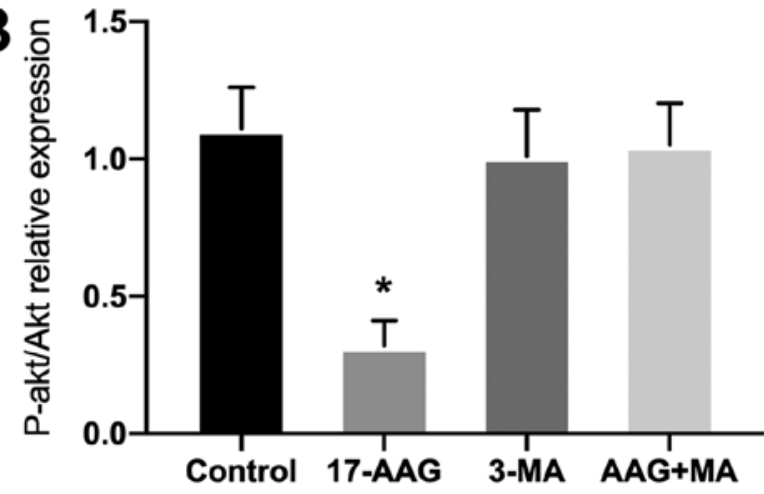

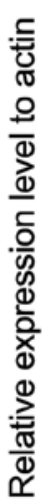
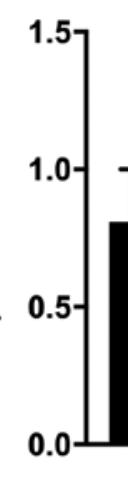
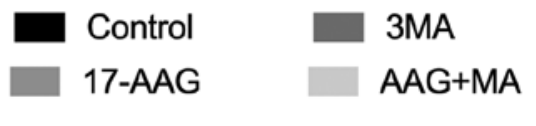

$\mathrm{AAG}+\mathrm{MA}$

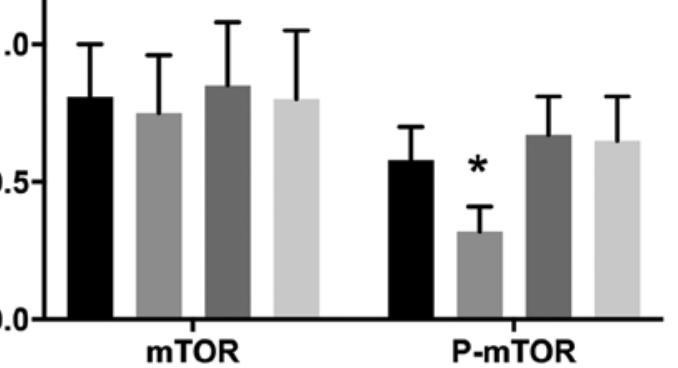

Figure 3. 17-AAG and/or 3-MA modulated Akt/mTOR signaling pathway. (A) Protein levels and (B) quantitative analysis of p-AKT and AKT. (C) Protein levels and (D) quantitative analysis of p-mTOR and mTOR. The data are presented as the mean \pm standard deviation. ${ }^{*} \mathrm{P}<0.05$ vs. control. $17-\mathrm{AAG} / \mathrm{AAG}$, 17-N-allylamino-17-demethoxygeldanamycin; 3-MA/MA, 3-methyladenine; p-phosphorylated.

To generate the incomplete ablation model, the present study used a laser fiber with a diameter of $300 \mu \mathrm{m}$ and a transplanted Huh7 tumor mouse to provide a model that can more easily measure molecular changes for subsequent studies (18). Our previous study (18) indicated that HSP90 inhibitors may promote apoptosis in the area of incomplete ablation, although an increase in efficiency was not observed. Another notable result is that 17-AAG not only induces apoptosis, but also activates autophagy in the residual tumor. Upon treatment with 17-AAG, a decreased level of LC3-I to LC3-II conversion was observed and a decrease in $\mathrm{p} 62$ protein levels, all of which are markers of autophagy activation.

The Akt/mTOR signaling pathway has emerged as the central conduit in the regulation of autophagy. Accumulating evidence has emphasized that the inhibition of Akt and its downstream target mTOR contributes to the initiation of autophagy (23-25). The present study assessed the Akt/mTOR pathway proteins using western blot analysis, which indicated that the 17-AAG group exhibited significantly decreased levels of p-Akt and p-mTOR expression with increased autophagy activity. In the group treated with a combination of 17-AAG and 3-MA, p-Akt and p-mTOR levels were not decreased and the corresponding increase in levels of autophagy was diminished. It could be hypothesized that this is due to a 3-MA-based inhibition of PI3K, which is important for a number of signaling pathways that control mTOR activation. 3-MA blocks class I PI3K persistently, whereas its suppressive effect on class III PI3K is transient. Class I PI3K is a heterodimer composed of p85-regulated and p110 catalytic subunits, resulting in AKT activation. Fully activated AKT leads to mTOR activation and the subsequent inhibition of autophagy. Although the possibility that other 17-AAG-mediated mechanisms may be responsible for the observed activation of autophagy cannot be completely excluded, accumulating evidence suggests that $\mathrm{Akt} / \mathrm{mTOR}$ inhibition is probably the mechanism of autophagy induction $(22,31)$.

An increasing body of evidence supports the existence of crosstalk between apoptosis and autophagy, including both positive and negative interactions (23-25). Recent evidence suggests that autophagy may attenuate drug-induced apoptotic responses $(31,32)$. In the present study, an increase in the activation of caspase- 3 was observed following treatment with 3-MA, which is a mark of apoptosis. Compared with treatment with 17-AAG alone, a combination of 17-AAG and 3-MA inhibited the increase of autophagy in a complimentary manner, resulting in a markedly enhanced level of apoptosis. To the best of our knowledge, this is the first study to highlight the interaction between apoptosis and autophagy in an animal model of residual tumors. This antagonism between autophagy and apoptosis can also be observed in an HCC incomplete ablation model, which suggests that the activation of autophagy has a protective effect on HCC cells and decreases the occurrence of apoptosis during incomplete ablation.

In summary, the results of the present study demonstrated that incomplete ablation and HSP90 inhibitor-induced autophagy involved enhanced autophagosomal synthesis and may negatively regulate apoptosis in Huh7 transplantation tumors. Therefore, autophagy has a pro-survival function in 
incompletely-ablated tumors treated with HSP90 inhibitors. Consistent with these results, the inhibition of autophagy may enhance the anti-cancer effects of HSP90 and therefore could be therapeutically targeted to improve treatment efficacy of combination therapy. In conclusion, the combined application of both drugs described in the present study has promise in clinical settings.

\section{Acknowledgements}

Not applicable.

\section{Funding}

This work was supported by Zhejiang Provincial Basic Public Welfare Research Program (grant no. LGD19C04007 to Fen Chen). The funders had no role in study design, data collection and analysis, decision to publish, or preparation of the manuscript.

\section{Availability of data and materials}

The datasets used and/or analyzed during the current study are available from the corresponding author on reasonable request.

\section{Authors' contributions}

SZ and FC conceived and designed the study. FC, HB and HX performed experiments. FC, HX and LV analyzed and interpreted the data. FC and LV drafted the manuscript. FC critically revised the manuscript for important intellectual content. FC performed statistical analysis. FC obtained funding. FC and HX provided technical or material support. SZ supervised the study. All authors read and approved the final manuscript.

\section{Ethics approval and consent to participate}

The protocol of this study was approved by the Research Ethics Committee of the First Affiliated Hospital, College of Medicine, Zhejiang University.

\section{Patient consent for publication}

Not applicable.

\section{Competing interests}

The authors declare that they have no competing interests.

\section{References}

1. Yun KC, Rhim H and Noh S: Radiofrequency ablation versus surgical resection as primary treatment of hepatocellular carcinoma meeting the Milan criteria: A systematic review. J Gastroenterol Hepatol 26: 1354-1360, 2011.

2. Hermida M, Cassinotto C, Piron L, Assenat E, Pageaux GP, Escal L, Pierredon-Foulongne MA, Verzilli D, Jaber S and Guiu B: Percutaneous thermal ablation of hepatocellular carcinomas located in the hepatic dome using artificial carbon dioxide pneumothorax: Retrospective evaluation of safety and efficacy. Int J Hyperthemia 35: 90-96, 2018.
3. Medhat E, Abdel Aziz A, Nabeel M, Elbaz T, Zakaria Z, Shousha H, Amer A, Fouad Fathalah W, Maher R and Musa S: Value of microwave ablation in treatment of large lesions of hepatocellular carcinoma. J Dig Dis 16: 456-463, 2015.

4. Schueller G, Kettenbach J, Sedivy R, Stift A, Friedl J, Gnant M and Lammer J: Heat shock protein expression induced by percutaneous radiofrequency ablation of hepatocellular carcinoma in vivo. Int J Oncol 24: 609-613, 2004.

5. Hinz S, Tepel J, Röder C, Kalthoff H and Becker T: Profile of serum factors and disseminated tumor cells before and after radiofrequency ablation compared to resection of colorectal liver metastases-a pilot study. Anticancer Res 35: 2961-2967, 2015.

6. Rai R, Richardson C, Flecknell P, Robertson H, Burt A and Manas DM: Study of apoptosis and heat shock protein (HSP) expression in hepatocytes following radiofrequency ablation (RFA). J Surg Res 129: 147-151, 2005.

7. Yang W, Ahmed M, Tasawwar B, Levchenko T, Sawant RR, Collins M, Signoretti S, Torchilin V and Goldberg SN: Radiofrequency ablation combined with liposomal quercetin to increase tumour destruction by modulation of heat shock protein production in a small animal model. Int $\mathbf{J}$ Hyperthermia 27: 527-538, 2011.

8. Schueller G, Kettenbach J, Sedivy R, Bergmeister H, Stift A, Fried J, Gnant M and Lammer J: Expression of heat shock proteins in human hepatocellular carcinoma after radiofrequency ablation in an animal model. Oncol Rep 12: 495-499, 2004.

9. He W, Ye X, Huang X, Lel W, You L, Wang L, Chen X and Qian W: Hsp90 inhibitor, BIIB021, induces apoptosis and autophagy by regulating mTOR-Ulk1 pathway in imatinib-sensitive and-resistant chronic myeloid leukemia cells. Int J Oncol 48: 1710-1720, 2016.

10. Leng AM, Liu T, Yang J, Cui JF, Li XH, Zhu YN, Xiong T, Zhang $G$ and Chen $Y$ : The apoptotic effect and associated signalling of HSP90 inhibitor 17-DMAG in hepatocellular carcinoma cells. Cell Biol Int 36: 893-899, 2012.

11. Liu X, Chen S, Tu J, Cai W and Xu Q: HSP90 inhibits apoptosis and promotes growth by regulating HIF-1 $\alpha$ abundance in hepatocellular carcinoma. Int J Mol Med 37: 825-835, 2016.

12. Liu X, Ban LL, Luo G, Li ZY, Li YF, Zhou YC, Wang XC, Jin CG, Ye JG, Ma DD, et al: Acquired resistance to HSP90 inhibitor 17-AAG and increased metastatic potential are associated with MUC1 expression in colon carcinoma cells. Anticancer Drugs 27: 417-426, 2016.

13. Chen Y, Youn P, Pysher TJ, Scaife CL and Furgeson DY: Tumour eradication using synchronous thermal ablation and Hsp90 chemotherapy with protein engineered triblock biopolymer-geldanamycin conjugates. Int J Hyperthermia 30: 550-564, 2014.

14. Lang SA, Moser C, Fichnter-Feigl S, Schachtschneider P, Hellerbrand C, Schmitz V, Schlitt HJ, Geissler EK and Stoeltzing O: Targeting heat-shock protein 90 improves efficacy of rapamycin in a model of hepatocellular carcinoma in mice. Hepatology 49: 523-532, 2009.

15. Webber PJ, Park C, Qui M, Ramalingam SS, Khuri FR, Fu H and $\mathrm{Du}$ Y: Combination of heat shock protein 90 and focal adhesion kinase inhibitors synergistically inhibits the growth of non-small cell lung cancer cells. Oncoscience 2: 765-776, 2015.

16. Yang R, Tang Q, Miao F, An Y,Li M, Han Y, Wang X, Wang J, Liu P and Chen R: Inhibition of heat-shock protein 90 sensitizes liver cancer stem-like cells to magnetic hyperthermia and enhances anti-tumor effect on hepatocellular carcinoma-burdened nude mice. Int J Nanomedicine 10: 7345-7358, 2015.

17. Moussa M, Goldberg SN, Kumar G, Sawant RR, Levchenko T, Torchilin V and Ahmed M: Radiofrequency ablation-induced upregulation of hypoxia-inducible factor- $1 \alpha$ can be suppressed with adjuvant bortezomib or liposomal chemotherapy. J Vasc Interv Radiol 25: 1972-1982, 2014.

18. Chen F, Bao H, Xie H, Tian G and Jiang T: Heat shock protein expression and autophagy after incomplete thermal ablation and their correlation. Int J Hyperthermia 36: 95-103, 2019.

19. Zhang R, Luo D, Miao R, Bai L, Ge Q, Sessa WC and Min W: Hsp90-Akt phosphorylates ASK1 and inhibits ASK1-mediated apoptosis. Oncogene 24: 3954-3963, 2005.

20. Sato S, Fujita N and Tsuruo T: Modulation of Akt kinase activity by binding to Hsp90. Proc Natl Acad Sci USA 97: 10832-10837, 2000.

21. Oral O, Akkoc Y, Bayraktar O and Gozuacik D: Physiological and pathological significance of the molecular cross-talk between autophagy and apoptosis. Histol Histopathol 31: 479-498, 2016. 
22. Heras-Sandoval D, Pérez-Rojas JM, Hernández-Damián J and Pedraza-Chaverri J: The role of PI3K/AKT/mTOR pathway in the modulation of autophagy and the clearance of protein aggregates in neurodegeneration. Cell Signal 26: 2694-2701, 2014.

23. Nazim UM and Park SY: Attenuation of autophagy flux by 6-shogaol sensitizes human liver cancer cells to TRAIL-induced apoptosis via p53 and ROS. Int J Mol Med 43: 701-708, 2019.

24. Mukhopadhyay S, Panda PK, Sinha N and Das DN, Bhutia SK: Autophagy and apoptosis: Where do they meet? Apoptosis 19: 555-566, 2014.

25. Mariño G, Niso-Santano M, Baehrecke EH and Kroemer G: Self-consumption: The interplay of autophagy and apoptosis. Nat Rev Mol Cell Biol 15: 81-94, 2014.

26. Talaei S, Mellatyar H, Asadi A Akbarzadeh A, Sheervalilou R and Zarghami N: Spotlight on 17-AAG as an Hsp90 inhibitor for molecular targeted cancer treatment. Chem Biol Drug Des 93: 760-786, 2019.

27. Walker AR, Klisovic R, Johnston JS, Jiang Y, Geyer S, Kefauver C, Binkley P, Byrd JC, Grever MR, Garzon R, et al: Pharmacokinetics and dose escalation of the heat shock protein inhibitor 17-allyamino-17-demethoxygeldanamycin in combination with bortezomib in relapsed or refractory acute myeloid leukemia. Leuk Lymphoma 54: 1996-2002, 2013.

28. Kaufmann SH, Karp JE, Litzow MR, Mesa RA, Hogan W, Steensma DP, Flatten KS, Loegering DA, Schneider PA, Peterson KL, et al: Phase I and pharmacological study of cytarabine and tanespimycin in relapsed and refractory acute leukemia. Haematologica 96: 1619-1626, 2011.
29. Heath EI, Hillman DW, Vaishampayan U, Sheng S, Sarkar F, Harper F, Gaskins M, Pitot HC, Tan W, Ivy SP, et al: A phase II trial of 17-allylamino-17-demethoxygeldanamycin in patients with hormone-refractory metastatic prostate cancer. Clin Cancer Res 14: 7940-7946, 2018.

30. Pacey S, Gore M, Chao D, Banerji U, Larkin J, Sarker S, Owen K, Asad Y, Raynaud F, Walton M, et al: A Phase II trial of 17-allylamino, 17-demethoxygeldanamycin (17-AAG, tanespimycin) in patients with metastatic melanoma. Invest New Drugs 30: 341-349, 2012.

31. Strozyk E and Kulms D: The role of AKT/mTOR pathway in stress response to UV-irradiation: Implication in skin carcinogenesis by regulation of apoptosis, autophagy and senescence. Int J Mol Sci 14: 15260-15285, 2013.

32. Janku F, Mcconkey DJ, Hong DS and Kurzrock R: Autophagy as a target for anticancer therapy. Nat Rev Clin Oncol 8: 528-539, 2011.

This work is licensed under a Creative Commons Attribution-NonCommercial-NoDerivatives 4.0 International (CC BY-NC-ND 4.0) License. 